\title{
Microsurgical Reconstruction of Receded Gingiva Using Alloderm In Esthetic Zone
}

\author{
Ranjana Mohan*, Robin Srivastava and Mohan Gundappa \\ Department of Periodontology, Teerthanker Mahaveer Dental College and Research Centre,India
}

Submission: July 14, 2017; Published: July 31, 2017

*Corresponding author: Ranjana Mohan, Department of Periodontology, Teerthanker Mahaveer Dental College and Research Centre, India, Email: Rajanamohan162@gmail.com

\begin{abstract}
Harmony of lips, gingival color, contour and the position, along with size, shape of teeth results in esthetically pleasing smile. Gingival recession in esthetic zone poses the greatest challenge to a clinician. Majority of periodontal plastic surgery are undertaken to treat or prevent marginal recession. Microsurgical reconstruction of the lost gingiva has been a recent advancement in periodontal plastic surgery. Patient aged 34 years reported to the department of Periodontology with the complaint of long looking upper corner tooth with sensitivity. Gingival recession with 13 was diagnosed and treated with minimally invasive periodontal plastic surgery for the root coverage using Alloderm with coronally advanced flap. $100 \%$ root coverage was obtained improving patients smile.
\end{abstract}

Keywords: Alloderm; Esthetics; Gingival recession; Minimally invasive microsurgery; Microsurgical reconstruction, Periodontal plastic surgery

Key Message: Successful management of gingival recession is the greatest challenge in esthetic zone. Recently introduced minimally invasive periodontal plastic surgery for localized gingival recession demonstrated wonderful results causing minimal surgical trauma with short duration of healing.

\section{Introduction}

Color, contour and shape of the gingival tissue greatly influence the esthetic of smile. Gingival shape is contingent upon the health of tissue, position of the free gingival margin and the volume and height of interdental papilla. Gingival architecture plays major role in creating smile. Conditions like gingival recession in esthetic zone poses challenge to a clinician. Gingival recession is defined as an apical displacement of soft tissues related to the cementoenamel junction [1].

Majority of periodontal plastic surgery are undertaken to treat or prevent marginal recession, ridge deficiency, and ridge collapse after extraction, excessive or asymmetrical gingival display and esthetic defects around implants.

Clinical goals of root coverage are to replace the lost tissue, reduce the sensitivity, improve the esthetics of the area when grafted tissue blend with the adjacent tissue color, texture and contour [1]. Recently, an Acellular dermal matrix graft has been used for root coverage [2-4].

Microsurgical technique has been a recent advancement in periodontal plastic surgery. The combination of small instruments and delicate surgical technique allows for extremely fine and accurate incisions, gentle tissue handling, and precise approximation of wound margins [5].

\section{Case History}

Patient aged 34 years reported to the department of Periodontology with the complaint of long corner tooth with sensitivity. On clinical examination, gingival recession was observed on maxillary right canine (Figure 1). Diagnosis was made as Miller's Class I gingival recession with 13. Coronally advanced flap with alloderm with microsurgical approach was planned. Patient's consent as well as ethical clearance was obtained prior to the surgical intervention. Following administration of local anesthesia, the tooth with the recession was root planed. A split-thickness flap with two vertical releasing incisions with micro scalpels was raised with microelevaters, and the papillae were de-epithelialized (Figure 2). Alloderm was measured, cut and rehydrated before suturing it with chromic gut 5-0 covering the defect as shown in Figure 3. The flap was coronally moved and secured to the de-epthelialized papillae over the alloderm with interrupted sutures (5-0 black braided silk) as shown in Figure 4. Pressure was applied before placing the periodontal dressing on surgical wound. Sutures were 
removed on 10th day and healing was found to be satisfactory (Figure 5). Healing is generally uneventful in minimally invasive surgery. Patient was evaluated after 3 months; there was $100 \%$ coverage of a denuded root with satisfactory gingival thickness and color match with microsurgical reconstruction of lost gingival (Figure 6).
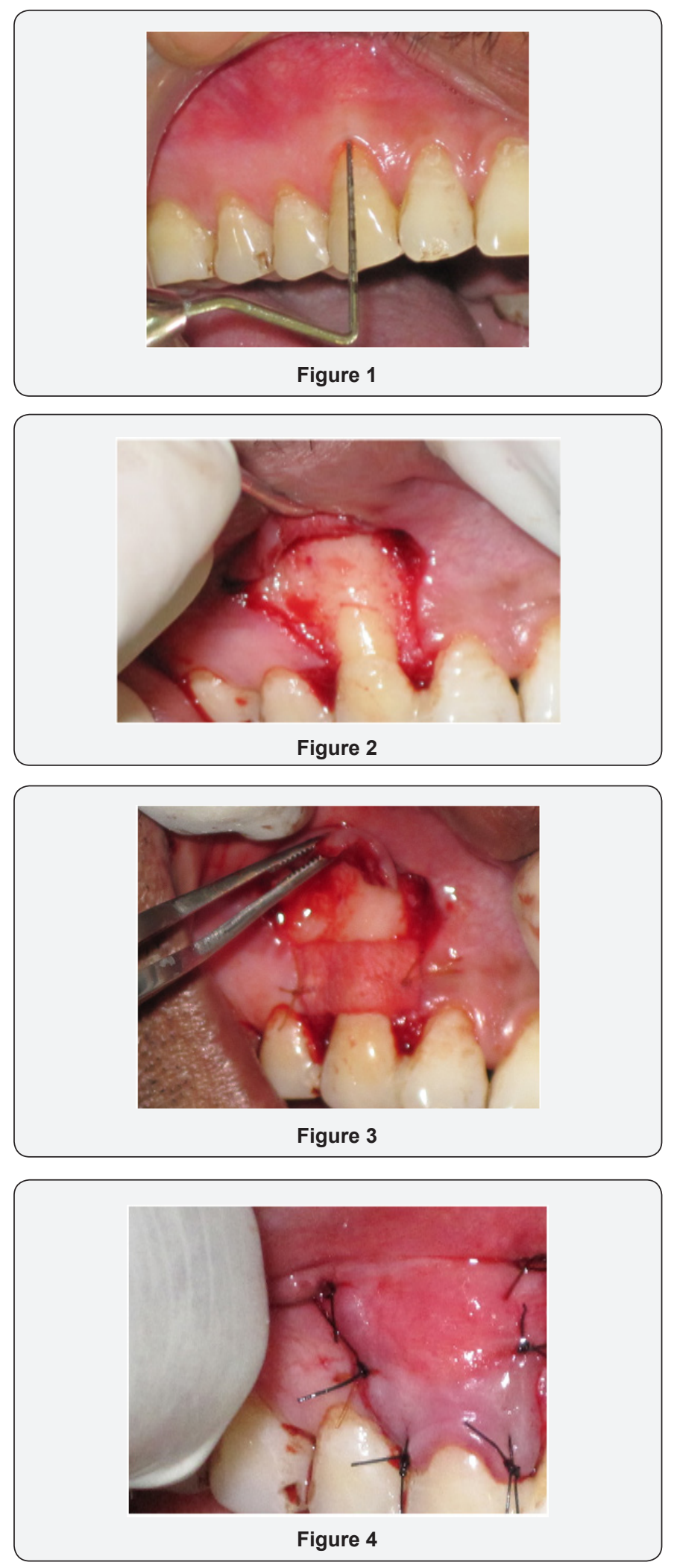
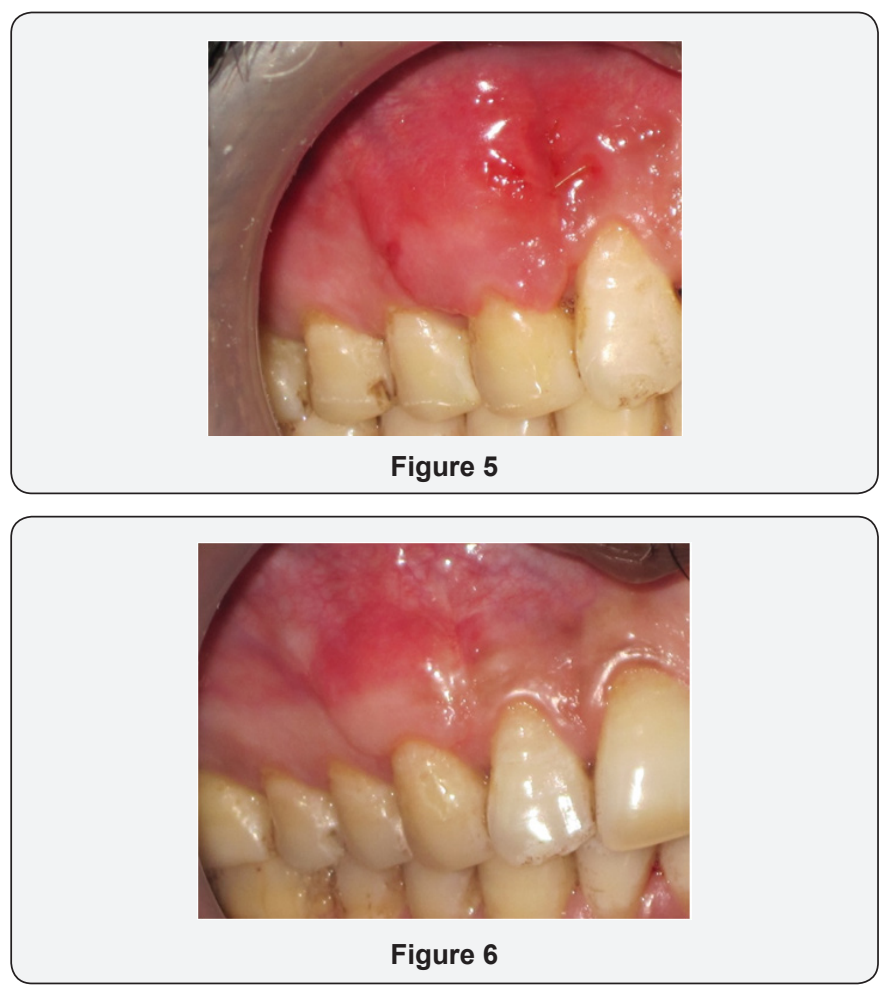

\section{Discussion}

Root exposure resulting from apical recession of marginal gingiva can create esthetic concerns for a patient. As the length of teeth increase, there is loss of gingival symmetry as well as increased sensitivity, susceptibility to caries and concern over retention of teeth. Restorative coverage of root can reduce sensitivity or treat caries but cannot decrease the length of clinical crown, restore lost periodontal support or prevent further gingival recession.

Various types of root coverage procedures are free autogenous graft, (gingival and connective tissue), pedicle flaps (lateral and coronal) and guided tissue regeneration with resorbable and non resorbable membranes. [1].

Pedicle flaps and Guided tissue regeneration are viable root coverage procedures but both techniques have limitations that reduce their clinical applicability. Nabers introduced the free gingival graft in 1966 and Sullivan and Atkins made further refinement in 1968. As originally described, graft was palatal keratinized mucosa (epithelium and connective tissue) approximately $1 \mathrm{~mm}$ thick. This type of graft was found to be unpredictable for covering roots due to sloughing of the grafted tissue over the avascular root surface. In the early 1980s, Miller and Holbrook \& Ochsenbein described the technique of thicker grafts with better predictability. However these grafts do not blend with the adjacent tissues and are readily identified as thicker and lighter in color. Moreover palatal wound remain exposed and heal with secondary intention. In 1985 Raetzke and later Langer and Langer described the use of connective tissue 
graft for root coverage. In this technique, epithelial component is eliminated from the graft and palatal connective tissue is transplanted over the recipient bed covered by gingival flap. The retained superior flap maintains the esthetics of the original tissue and acts as a source of epithelial cells migrating over the connective tissue graft. These grafts are very successful for root coverage and blending with the adjacent tissue producing high esthetic results. Several modifications of this technique have been proposed and connective tissue grafts are considered the gold standard for treatment of gingival recession [6-8].

However, this technique involves a certain degree of discomfort to the patient because an additional palatal donor site has to be prepared, increasing the risk of postoperative pain and hemorrhage. Recently, an Acellular dermal matrix graft has been used as a substitute for the palatal donor sites to increase the width of keratinized tissue around the teeth and implants and for root coverage procedures [9-11]. Processing of the dermis obtained from human donor removes all cells, leaving a structurally intact connective tissue matrix composed of type-I collagen. Harris [9] reported the use of this acellular dermal matrix graft with coronally positioned flap for the treatment of gingival recession. The Acellular dermal matrix consistently integrated into the host tissue, maintaining structural integrity of the tissue and revascularized via preserved vascular channels. The color match obtained was also reported to be comparable to that of the connective tissue graft.

Microsurgical reconstruction with minimally invasive technique is performed with magnification and microsurgical instruments and suture materials. Combination of small instruments and delicate surgical technique allows for extremely fine and accurate incisions, gentle tissue handling, and precise approximation of wound margins [1].

\section{Conclusion}

Present case report is the excellent example of root coverage using Alloderm for the management of gingival recession with coronally advanced flap. Minimally invasive approach using magnification and microsurgical instruments has demonstrated wonderful results due to minimal tissue trauma and reduced healing time. Minimal intervention periodontal plastic surgery especially in esthetic zone significantly contributes to improve the smile in short span of time.

\section{References}

1. Pasquinelli K (1999) Periodontal plastic surgery. J Can Den Asso 75(3): 203-203.

2. Callan PP (1996) Use of acellular dermal matrix allograft material in dental implant treatment. Dent Surg Products 9(2): 14-7.

3. Silverstein LH, Callan DP (1996) An acellular dermal matrix allograft substitute for palatal donor tissue. Postgrad Dent 19(2): 14-21.

4. Batista EL, Batista FC, Novars A Jr (2001) Management of soft tissue ridge deformities with acellular dermal matrix.Clinical approach and outcome after 6 months of treatment. J Periodontal 72(2):265-73.

5. Burkhardt R (2000) Utilization of the surgical microscope for advanced plastic surgery. Pract Periodont Aesthet Dent 12(2): 171-180.

6. Raetzke PB (1985) Covering localized areas of root exposures employing the envelope technique. J Periodontal 56(7): 397- 402.

7. Nelson SW (1987) The subepithelial connective tissue graft, a bilaminar reconstructive procedure for root coverage of denuded root surfaces. J Periodontal 58(2): 94-102.

8. Bouchard P, Etienne D, Ouhayoun JP (1994) Nilvens R Sub-epithelial connective tissue graft in the treatment of gingival recession - A comparative study of 2 procedures. J Periodontal 65(11): 929-36.

9. Harris RJ (1998) Root coverage with a connective tissue with partial thickness double pedicle graft and an acellular dermal matrix: A clinical and histlogical evaluation of a case report. J Periodontal 69(11): 1305-1311.

10. Aichelmann-Reidy ME, Yukna RA, Mayer ET(1999) Acellular dermal matrix used for root coverage. J Periodontal 34(6): 70-223.

11. Harris RJ (2000) A comparative study of root coverage obtained with an acelluar dermal matrix versus a connective tissue graft. Results of 107 recession defects in 50 consecutively treated patients. Int J Periodontics Restorative Dent 20(1): 51-59.

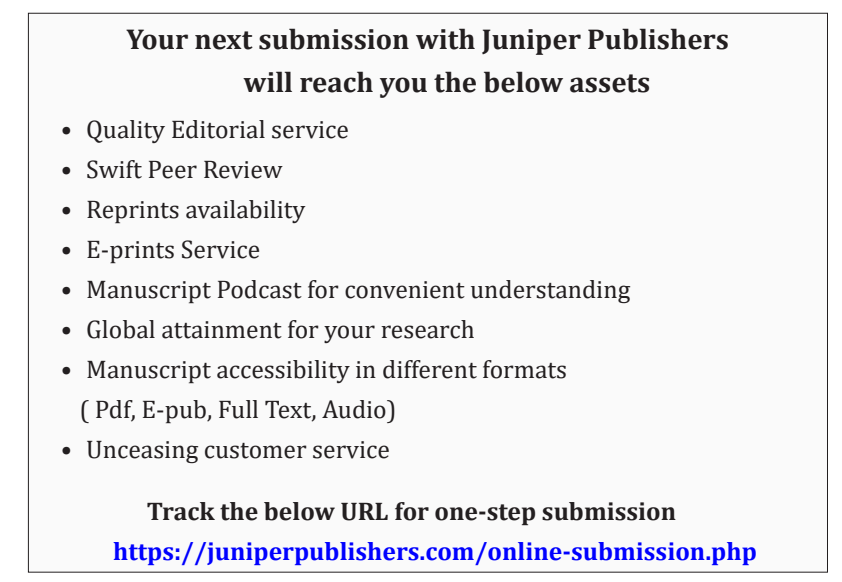

